\title{
Impact of Lavender on Pain and Anxiety Levels Associated With Spine Procedures
}

\author{
Maria Grabnar, MD, ${ }^{1}$ Mary Joan Roach, PhD, ${ }^{1}$ Alaa Abd-Elsayed, MD, MPH, ${ }^{2}$ Chong Kim, MD ${ }^{1}$ \\ ${ }^{1}$ Department of Physical Medicine and Rehabilitation, Case Western Reserve University/MetroHealth, Cleveland, $\mathrm{OH}^{2}$ Department of \\ Anesthesiology, University of Wisconsin School of Medicine and Public Health, Madison, WI
}

\begin{abstract}
Background: To reduce pain and anxiety associated with interventional pain procedures, sedation is often used, with benzodiazepines, opioids, and propofol the most commonly used classes of drugs for sedation. However, patient coherence and ability to communicate procedural pain and abnormal sensations help prevent adverse outcomes. Therefore, discovering alternative therapies to mitigate the anxiety and pain associated with these procedures and to minimize risk is important. The aim of our study was to investigate whether lavender has an effect on pain and anxiety associated with lumbar epidural steroid injections and lumbar medial branch blocks.

Methods: In this randomized controlled study, 54 subjects were randomly assigned to 1 of 3 intervention groups, and 46 patients were included in the final analysis: experimental lavender group $(n=17)$, control almond oil group $(n=15)$, and placebo sterile water group $(n=14)$. Patients wore a mask infused with either lavender, almond oil, or water for 5 minutes prior to and during their procedure. Patients rated their anxiety using the State-Trait Anxiety Inventory prior to and after the procedure based on how they felt during the procedure. Patients rated their pain according to the numerical rating scale. Outcome measures were a comparison of pain among the 3 groups and a comparison of the change in anxiety before and after the procedure among the 3 groups.

Results: The lavender group demonstrated the highest mean change in anxiety scores (9.9) compared to almond oil (5.3) and water (3.6) preprocedurally vs postprocedurally. The lavender group also reported the lowest mean pain level (3.8) compared to almond oil (5.6) and water (5.6). However, none of the differences between groups showed statistical significance at the $P<0.05$ level.

Conclusion: Lavender may have a clinically beneficial effect on anxiety levels and pain reduction.
\end{abstract}

Keywords: Anti-anxiety agents, aromatherapy, lavandula, pain management

Address correspondence to Chong Kim, MD, Department of Physical Medicine and Rehabilitation, Case Western Reserve University/MetroHealth, 2500 MetroHealth Dr., Cleveland, OH 44109. Tel: (216) 778-7800. Email: ckim3@metrohealth.org

\section{INTRODUCTION}

Back pain is one of the most common forms of musculoskeletal pain causing patients to seek medical care cat, $^{1,2}$ and is the leading cause of activity limitation and work loss throughout most of the world. ${ }^{3}$ Low back pain has a lifetime prevalence between $49 \%$ and $90 \%$; therefore, most individuals will experience this condition at some point. ${ }^{4}$ Back pain secondary to spinal disease can be a challenging clinical condition to treat and can have several different etiologies. Based on evidence-based clinical practice guidelines for interventional techniques, ${ }^{5}$ the evidence for diagnostic lumbar facet joint nerve blocks is good, with $75 \%$ to $100 \%$ pain relief as the criterion standard. The same guidelines found good evidence for lumbar epidurals in managing disc herniation or radiculitis; fair evidence for managing axial or discogenic pain without disc herniation, radiculitis, or facet joint pain; and fair evidence for treating postsurgery syndrome with caudal epidural injections.
Interventional spine procedures such as lumbar epidural steroid injections and nerve blocks can be particularly painful or anxiety-provoking for patients. Introduction of needles can cause patient discomfort because of the presence of nociceptors in skin and underlying tissue. ${ }^{6}$ Studies have shown a correlation between pain and anxiety. ${ }^{7-9}$ To reduce pain and anxiety associated with interventional pain procedures, sedation is often used. Based on a 2017 survey that involved 337 pain physicians, sedation is used in approximately $50 \%$ of epidural injections and medial branch blocks. ${ }^{10}$ The survey also found that the most commonly used classes of drugs for sedation were benzodiazepines (97\% of respondents), opioids (77\% of respondents), and propofol (36\% of respondents). Today's medical culture places an emphasis on patient satisfaction, which may be a reason that sedation is frequently used. However, studies have shown a lack of significant improvement in patient satisfaction with the use of sedation. ${ }^{11}$ The use of anxiolytics 
and analgesics carries a significant amount of risk involving erroneous nerve ablation and local anesthetic systemic toxicity; therefore, patient alertness during procedures involving spinal nerves is imperative. Patient coherence and ability to communicate procedural pain and abnormal sensations help prevent adverse outcomes. ${ }^{12}$ The American Society of Regional Anesthesia and Pain Medicine convened a group of experts to develop the Practice Advisory on Neurologic Complications in Regional Anesthesia and Pain Medicine, and per the guidelines, general anesthesia or heavy sedation removes the opportunity for the patient and/or physician to recognize, report, and respond to an atypical symptom during the interventional spine procedure, and the guidelines therefore recommend against this practice. ${ }^{12,13}$ In addition to impairing patient alertness during procedures, short-acting anxiolytic agents such as benzodiazepines and pain-relieving opiates are associated with side effects such as respiratory depression ${ }^{14,15}$ and may skew medial branch block outcomes. Benzodiazepines produce skeletal muscle relaxation and amnesia that may result in a false-positive response by alleviating baseline back pain ${ }^{16}$ or in a false-negative response by reducing pain tolerance. ${ }^{17}$ Therefore, discovering alternative therapies to mitigate the anxiety and pain associated with these procedures and to minimize risk and false outcomes is important.

Patients often use aromatherapy for pain relief, psychological comfort, and disease prevention, although evidence for the therapeutic efficacy of aromatherapy remains limited. ${ }^{18}$ Aromatherapy is defined as the therapeutic use of essential oils derived from plants and involves transmission of signals from the olfactory system to the brain, which regulates anxiety, depression, and mood disorders by secreting neurotransmitters such as serotonin and dopamine. ${ }^{19}$ While more than 40 plant derivatives have been identified for therapeutic use, lavender, eucalyptus, rosemary, chamomile, and peppermint are the most frequently used extracts. ${ }^{20}$

Lavender extracts are widely used in aromatherapy. Lavender aromatherapy has been reported to possess several therapeutic effects including antianxiety, mood stabilization, and analgesia. ${ }^{21}$ Lavender essential oil is primarily sold as an over-the-counter herbal medicine and has been granted the status of "Generally Recognized as Safe" by the US Food and Drug Administration, an indication of its safety when used as a dietary supplement. ${ }^{22}$

Clinically, lavender has been shown to decrease anxiety in patients scheduled to undergo colorectal surgery ${ }^{23}$ and has been shown to decrease anxiety associated with dental procedures. ${ }^{24}$ Lavender has also been shown to decrease pain during procedures, particularly when inhaled prior to a generally painful procedure such as catheter or needle insertion and chest tube removal. ${ }^{25-27}$ While the effects of lavender on various medical procedures have been investigated, no studies to the authors' knowledge have examined the effects of lavender on fluoroscopic spine procedures. This study investigated whether inhalation of lavender essential oil reduced the pain and anxiety associated with interventional spine procedures, particularly lumbar epidural injections and medial branch blocks.

\section{METHODS}

This study was approved by the MetroHealth Institutional Review Board. In addition, this study was registered at clinicaltrials.gov (NCT04257019). Clinical consent for the procedure was first obtained and then the subjects were consented for the study. After informed written consent was obtained, 56 patients $\geq 21$ years who presented for lumbar epidural or lumbar medial branch block were included in the study. Exclusion criteria included an allergy or sensitivity to lavender or almond oil, a preexisting problem with the sense of smell, the inability to follow basic instructions relating to the design of the experiment, or the inability to answer questions regarding their pain or anxiety. Subjects were assigned to each group by a computer-generated randomizer.

The method of inhalation involved instillation of either lavender (100\% Lavandula $x$ intermedia [PipingRock Health Products]), sweet almond oil (100\% Prunus amygdalus dulcis [PipingRock Health Products]), or sterile water into a standard surgical mask. Five drops were applied to different areas on the interior of the mask prior to asking the subject to don the mask on the day of the scheduled procedure.

After consent was obtained, basic demographic data were collected by medical record review and brief interview with the patient, including the procedure type, whether the patient took an anxiolytic or analgesic on that day, and whether the patient had had a lumbar epidural injection or medial branch block performed during the preceding 2 years. Patients were then asked to complete the State-Trait Anxiety Inventory (STAI) questionnaire regarding their current anxiety. The STAI is a psychological inventory based on a 4-point Likert scale and consists of 20 questions with total scores ranging from 20 to 80; higher scores indicate higher anxiety.

Patients were then taken to a quiet area away from the triage room, asked to put on a standard surgical mask, and instructed to breathe slowly and deeply for 5 minutes. Patients were then taken to the fluoroscopy suite for their scheduled procedure. Patients were told that they could remove their mask and terminate participation in the study at any point without any effect on the procedure. Immediately after completion of the procedure, patients were asked to remove the mask, taken back to the triage area, and asked to complete the STAI questionnaire again based on how they felt during the procedure. In addition, subjects were asked to rate their pain on the numerical rating scale (NRS) based on the pain they experienced during the procedure. The scale ranges from 0 to 10 , with 0 being least severe and 10 being most severe.

The authors intended to recruit 75 subjects to have sufficient power, but the study had to be stopped because of critical mask shortages during the coronavirus disease 2019 (COVID-19) pandemic.

As stated earlier, 56 subjects were initially recruited, but 2 withdrew from the study. One subject in the lavender group reported that when filling out the STAI questionnaire, she became upset as it made her reflect on personal life events, and she requested to be withdrawn from the study. Another subject reported that she felt claustrophobic with the surgical mask on and also requested to be withdrawn from the study.

For the statistical analysis, only patients who answered all the questions were included, which was 46 of the 54 patients. Of those 46 patients, 6 took an anxiolytic (benzodiazepine-alprazolam, clonazepam, or diazepam) on the day of the procedure; none reported taking an analgesic. Patients were told that benzodiazepines, opioids, or 


\begin{tabular}{lccc} 
Variable & Lavender, $\mathbf{n = 1 7}$ & Almond Oil, $\mathbf{n = 1 5}$ & Water, $\mathbf{n = 1 4}$ \\
\hline Sex & & $5(33.3)$ & $4(29.6)$ \\
$\quad$ Male & $8(47.0)$ & $10(66.7)$ & $10(71.4)$ \\
$\quad$ Female & $9(53.0)$ & 57.1 & 52.6 \\
Mean age, years & 54.2 & $6(40.0)$ & $5(35.7)$ \\
Prior procedure & $13(76.5)$ & - & $4(28.6)$ \\
Anxiolytic or analgesic prior to the procedure & $2(11.8)$ & & \\
\hline
\end{tabular}

Note: Values are $n(\%)$ unless otherwise indicated.

nonsteroidal anti-inflammatory drugs were included in these categories. We did not ask patients to report scheduled daily medications such as selective serotonin reuptake inhibitors and anticonvulsants.

The 46 patients were randomized into the following treatment groups: 17 in the lavender group, 15 in the almond oil group, and 14 in the water group.

Outcome measures were comparison of pain among the 3 groups during the procedure and comparison of change in anxiety among the 3 groups before and after the procedure using analysis of variance. Statistical analysis was performed using SPSS software, version 26 (IBM Corp).

\section{RESULTS}

Table 1 shows descriptive characteristics among groups. Mean age was similar among groups. All 3 groups had a higher percentage of females compared to males, but the differences were not statistically significant.

Figure 1 shows the pain level during the procedure among groups. Subjects in the lavender group showed a lower mean pain score (3.8) during the procedure than subjects in both the almond oil (5.6) and water (5.6) groups. Table 2 shows the between-group differences for pain levels. The betweengroup difference for lavender compared to both water and almond oil was -1.8 , and the $95 \% \mathrm{Cl}$ did not cross 0 .

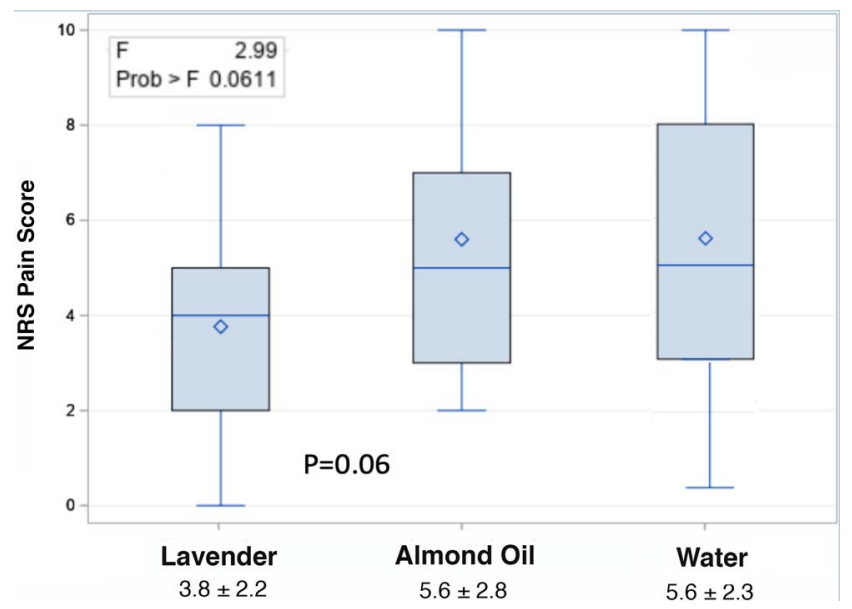

Figure 1. Pain scores during procedures by treatment group. NRS, numerical rating scale.
Figure 2 shows the change in anxiety preprocedure vs postprocedure by treatment group. Subjects in the lavender group showed a greater reduction in anxiety scores (9.9) compared to the almond oil (5.3) and water (3.6) groups. Table 3 shows the between-group differences for the change in anxiety scores.

None of the differences between groups showed statistical significance at the $P<0.05$ level.

We performed a separate analysis to check for confounding variables. Whether subjects took anxiolytics or analgesics on the day of the scheduled procedure or had the same procedure during the preceding 2 years had no effect on results.

No adverse reactions to lavender or almond oil were reported, but 3 subjects reported that the smell was potent in the lavender group.

\section{DISCUSSION}

This study focused on the anxiolytic and analgesic effects of lavender using 2 questionnaires, the NRS for pain and the STAI for anxiety, in patients scheduled for lumbar epidural injections and lumbar medial branch blocks. Inhalation of lavender resulted in a greater change in anxiety scores after wearing a mask infused with lavender when compared to the almond oil and placebo groups, but this change was not statistically significant. Based on a 2019 systematic review, 54 of 65 randomized controlled trials reported a significant result in favor of lavender use for anxiety when measured by the STAI: a mean change of $-5.99(95 \% \mathrm{Cl}-9.39$ to -2.59 ; $P<0.001){ }^{28}$ Inhalation of lavender also resulted in lower pain

Table 2. Differences in Pain Scores Between Treatment Groups During Procedures

\begin{tabular}{lcc}
\hline Comparison & $\begin{array}{c}\text { Difference } \\
\text { Between Groups }\end{array}$ & $\mathbf{9 5 \%} \mathbf{~ C l}$ \\
\hline Lavender vs water & -1.8 & -3.6 to -0.03 \\
Lavender vs almond oil & -1.8 & -3.6 to -0.09 \\
Almond oil vs water & -0.3 & -1.8 to -1.9 \\
\hline
\end{tabular}

Note: Mean pain scores given in Figure 1 are rounded, resulting in the same pain score (5.6) for the almond oil and water groups. The difference between these groups, however, was calculated based on the full average pain score, and the difference between the two groups was -0.3 as shown in Table 2. 


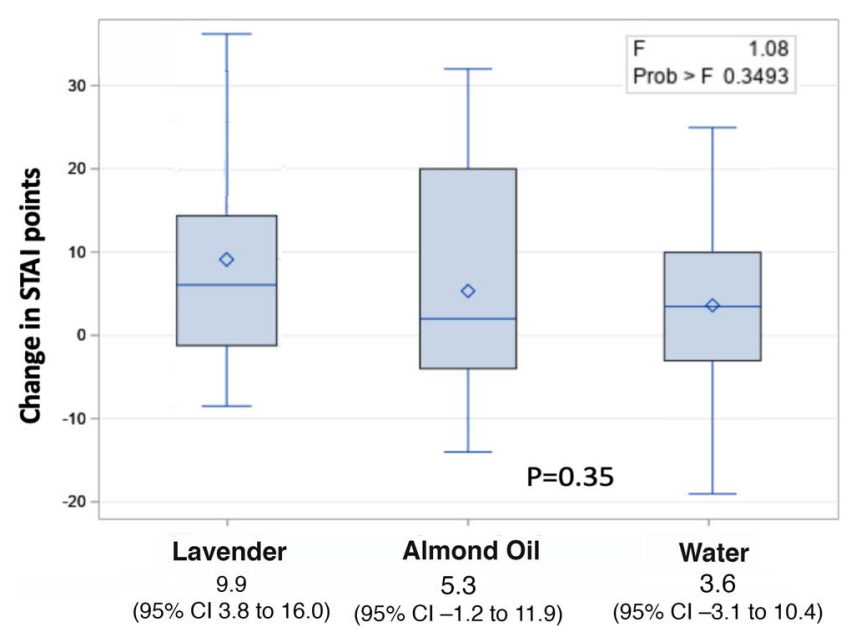

Figure 2. Change in anxiety scores preprocedure vs postprocdure by treatment group. STAI, State-Trait Anxiety Inventory.

levels experienced during the scheduled procedure when compared to almond oil and placebo at a clinically relevant although not statistically significant level. Based on studies evaluating clinical meaningfulness for reduction in pain among patients with low back pain, a score of 1.8 to 1.9 on the NRS that was used in our study correlates with a minimum clinically important change. ${ }^{29}$

Different mechanisms have been proposed for the effects of lavender therapy. First is the influence of aroma on the brain through the limbic system via olfaction, and the other is through the direct pharmacologic effects of the essential oil. Lavender contains 2 compounds, linalool and linalyl acetate, both of which have been shown to stimulate the parasympathetic nervous system. ${ }^{30}$ The anxiolytic effects of lavender may be attributable to the antagonizing effects on the N-methyl-D-aspartate receptor and inhibition of the serotonin transporter, which may explain its antiagitation effect in animals. ${ }^{31}$ Another mechanism proposed is the effect of lavender on the serotonin receptor in specific areas of the brain (hippocampus, anterior cingulate cortex, temporal gyrus, insula) through a reduction of its expression and binding ability, the way that a selective serotonin receptor inhibitor medication works. ${ }^{32}$ In animal models, linalyl acetate anxiolytic effects were not observed in anosmic mice, indicating that the effects were triggered by olfactory input. In addition, flumazenil, a benzodiazepine antagonist that competitively inhibits by interacting with benzodiazepine receptor sites on the GABA/benzodiazepine receptor complex, was found to antagonize odor-induced

Table 3. Differences in Anxiety Scores Between Treatment Groups Preprocedure vs Postprocedure

\begin{tabular}{lcc}
\hline Comparison & $\begin{array}{c}\text { Difference } \\
\text { Between Groups }\end{array}$ & $\mathbf{9 5 \% ~ C l}$ \\
\hline Lavender vs water & -6.3 & -15.4 to 2.8 \\
Lavender vs almond oil & -4.6 & -13.5 to 4.3 \\
Almond oil vs water & -1.7 & -11.0 to 7.7 \\
\hline
\end{tabular}

anxiolytic effects, indicating that gamma-aminobutyric acid transmission plays an important role in anxiolytic effects. ${ }^{33}$

Other studies have investigated inhalation of essential oils during interventional spine procedures. Eucalyptus, specifically its constituent 1,8-cineole, was found to decrease anxiety before selective nerve root blocks. ${ }^{34} \mathrm{~A}$ meta-analysis conducted in 2016 summarized 12 studies that examined the effects of aromatherapy on pain, and a significant positive effect was found in the reduction of pain. Types of aromatherapy included in the studies were lavender, eucalyptus, lemon grass, and rose oil. ${ }^{35}$

The potential for anxiety to alter the course of pain is well known. Evidence shows that the emotional and behavioral responses to pain are strongly guided by 2 related psychosocial factors, fear and anxiety. Individuals who have lower anxiety do not interpret pain as negatively as those with higher anxiety levels, which aids in recovery. ${ }^{36}$ In addition, satisfaction with pain management often has little correlation to pain reduction and is more often associated with communication, staff behavior, and empathy. ${ }^{37}$ A pleasant scent added to a potentially anxiety-provoking experience, such as a fluoroscopically guided medical procedure, may play a role in patient satisfaction.

This study has several limitations. The study administrator was not blinded to which patients received which treatment, and although patients were told they were assigned to 1 of 3 groups and not told which treatment they were receiving, complete blinding, because of the smell of the lavender and almond oil, was not possible. These results are based on a preliminary analysis because of the COVID-19 pandemic that resulted in a temporary pause in all clinical research due to public health guidelines and a shortage of masks that limited the sample size and the duration of the study. Future directions include conducting a larger-scaled study and expanding the procedure pool to include other fluoroscopically guided procedures such as cervical epidurals and cervical medial branch blocks as well as genicular nerve blocks and radiofrequency ablation. As some subjects reported that the smell of lavender was potent, researchers for future studies may consider using a dilution or instilling $<5$ drops of lavender into a mask. Last, whether instructing patients to breathe slowly and deeply had an effect on outcomes, because all patients were asked to breathe this way, is unknown.

Because of patient tolerance of the intervention, lack of adverse effects, low cost, and clinical effect on pain and anxiety levels, a feasible clinical practice may be to include a lavender scent in the waiting area or preprocedural area.

\section{CONCLUSION}

Lavender may have a clinically meaningful effect on decreasing pain during lumbar epidural injections and medial branch blocks but was not found to have a significant effect on reducing anxiety related to lumbar epidural injections and medial branch blocks.

\section{ACKNOWLEDGMENTS}

The authors thank Richard Wilson, MD for guidance with the study design and analysis and Jayme Knutson, PhD for calculating an estimate of the power of the study. The authors have no financial or proprietary interest in the subject matter of this article. 


\section{REFERENCES}

1. Bell JA, Burnett A. Exercise for the primary, secondary and tertiary prevention of low back pain in the workplace: a systematic review. J Occup Rehabil. 2009;19(1):8-24. doi: 10.1007/s10926-009-9164-5

2. Walker BF. The prevalence of low back pain: a systematic review of the literature from 1966 to 1998. J Spinal Disord. 2000;13(3):205-217. doi: 10.1097/00002517-200006000-00003

3. Lidgren L. The bone and joint decade 2000-2010: an update. Acta Orthop Scand. 2000;71(1):3-6. doi: $10.1080 / 00016470052943810$

4. Scott NA, Moga C, Harstall C. Managing low back pain in the primary care setting: the know-do gap. Pain Res Manag. 2010;15(6):392-400. doi: 10.1155/2010/252695

5. Manchikanti L, Abdi S, Atluri S, et al. An update of comprehensive evidence-based guidelines for interventional techniques in chronic spinal pain. Part II: guidance and recommendations. Pain Physician. 2013;16(2 Suppl):S49-S283.

6. Rice FL, Albrecht PJ. Cutaneous mechanisms of tactile perception: morphological and chemical organization of the innervation to the skin. In: Masland RH, Albright TD, Gardner EP (eds). The Senses: A Comprehensive Reference. New York: Elsevier: 2008. doi: 10.1016/b978-012370880-9.00340-6

7. Beesdo K, Hoyer J, Jacobi F, Low NCP, Höfler M, Wittchen HU. Association between generalized anxiety levels and pain in a community sample: evidence for diagnostic specificity. $J$ Anxiety Disord. 2009;23(5):684-693.

doi: 10.1016/j.janxdis.2009.02.007

8. Sanikop S, Agrawal P, Patil S. Relationship between dental anxiety and pain perception during scaling. J Oral Sci. 2011;53(3):341-348. doi: 10.2334/josnusd.53.341

9. Patout $\mathrm{M}$, Lamia B, Lhuillier $\mathrm{E}$, et al. A randomized controlled trial on the effect of needle gauge on the pain and anxiety experienced during radial arterial puncture. PLoS One. 2015;10(9):e0139432. doi: 10.1371/journal.pone.0139432

10. Kohan L, Salajegheh R, Hamill-Ruth RJ, Yerra S, Butz J. A review and survey of policies utilized for interventional pain procedures: a need for consensus. J Pain Res. 2017;10:625-634. doi: 10.2147/JPR.S126851

11. Diehn FE, Geske JR, Amrami KK, et al. An audit of transforaminal epidural steroid injections without sedation: low patient dissatisfaction and low vasovagal rates. Pain Med. 2013;14(7):994-998. doi: 10.1111/pme.12092

12. Neal JM, Bernards CM, Hadzic A, et al. ASRA practice advisory on neurologic complications in regional anesthesia and pain medicine. Reg Anesth Pain Med. 2008;33(5):404-415.

13. Neal JM, Barrington MJ, Brull R, et al. The second ASRA practice advisory on neurologic complications associated with regional anesthesia and pain medicine: executive summary 2015. Reg Anesth Pain Med. 2015;40(5):401-430. doi: 10.1097/AAP.0000000000000286

14. Leino K, Mildh L, Lertola K, Seppälä T, Kirvelä O. Time course of changes in breathing pattern in morphine- and oxycodone-induced respiratory depression. Anaesthesia. 1999;54(9):835-840. doi: 10.1046/j.1365-2044.1999.00946.x

15. Henderson A, Wright M, Pond SM. Experience with 732 acute overdose patients admitted to an intensive care unit over six years. Med J Aust. 1993;158(1):28-30. doi: 10.5694/j.1326-5377.1993.tb121644.x

16. Chou R. Pharmacological management of low back pain. Drugs. 2010;70(4):387-402. doi: 10.2165/11318690-000000000-00000

17. Frölich MA, Zhang K, Ness TJ. Effect of sedation on pain perception. Anesthesiology. 2013;118(3):611-621. doi: 10.1097/ALN.0b013e318281592d
18. Zhang $Y$, Wu Y, Chen T, et al. Assessing the metabolic effects of aromatherapy in human volunteers. Evid Based Complement Alternat Med. 2013;2013:356381. doi: $10.1155 / 2013 / 356381$

19. Lv XN, Liu ZJ, Zhang HJ, Tzeng CM. Aromatherapy and the central nerve system (CNS): therapeutic mechanism and its associated genes. Curr Drug Targets. 2013;14(8):872-879. doi: $10.2174 / 1389450111314080007$

20. Boehm K, Büssing A, Ostermann T. Aromatherapy as an adjuvant treatment in cancer care-a descriptive systematic review. Afr J Tradit Complement Altern Med. 2012;9(4):503-518. doi: 10.4314/ajtcam.v9i4.7

21. Cavanagh HMA, Wilkinson JM. Biological activities of lavender essential oil. Phytother Res. 2002;16(4):301-308. doi: 10.1002/ptr.1103

22. Code of Federal Regulations Title 21. U.S. Food and Drug Administration. September 21, 2021. Accessed September 29, 2021. ecfr.gov/current/title-21

23. Ayik C, Özden D. The effects of preoperative aromatherapy massage on anxiety and sleep quality of colorectal surgery patients: a randomized controlled study. Complement Ther Med. 2018;36:93-99. doi: 10.1016/j.ctim.2017.12.002

24. Zabirunnisa M, Gadagi JS, Gadde P, Myla N, Koneru J, Thatimatla C. Dental patient anxiety: possible deal with lavender fragrance. J Res Pharm Pract. 2014;3(3):100-103. doi: 10.4103/2279-042X.141116

25. Yayla EM, Ozdemir L. Effect of inhalation aromatherapy on procedural pain and anxiety after needle insertion into an implantable central venous port catheter: a quasi-randomized controlled pilot study. Cancer Nurs. 2019;42(1):35-41. doi: 10.1097/NCC.0000000000000551

26. Bagheri-Nesami M, Espahbodi F, Nikkhah A, Shorofi SA, Charati $J Y$. The effects of lavender aromatherapy on pain following needle insertion into a fistula in hemodialysis patients. Complement Ther Clin Pract. 2014;20(1):1-4. doi: 10.1016/j.ctcp.2013.11.005

27. Hasanzadeh F, Kashouk NM, Amini S, et al. The effect of cold application and lavender oil inhalation in cardiac surgery patients undergoing chest tube removal. EXCLIJ. 2016;15:64-74. doi: 10.17179/excli2015-748

28. Donelli D, Antonelli M, Bellinazzi C, Gensini GF, Firenzuoli F. Effects of lavender on anxiety: a systematic review and meta-analysis. Phytomedicine. 2019;65:153099. doi: 10.1016/j.phymed.2019.153099

29. Hägg O, Fritzell P, Nordwall A; Swedish Lumbar Spine Study Group. The clinical importance of changes in outcome scores after treatment for chronic low back pain. Eur Spine J. 2003;12(1):12-20. doi: 10.1007/s00586-002-0464-0

30. Duan X, Tashiro M, Wu D, et al. Autonomic nervous function and localization of cerebral activity during lavender aromatic immersion. Technol Health Care. 2007;15(2):69-78.

31. López V, Nielsen B, Solas M, Ramírez MJ, Jäger AK. Exploring pharmacological mechanisms of lavender (Lavandula angustifolia) essential oil on central nervous system targets. Front Pharmacol. 2017;8:280. doi: 10.3389/fphar.2017.00280

32. Baldinger $P$, Höflich AS, Mitterhauser $M$, et al. Effects of silexan on the serotonin-1A receptor and microstructure of the human brain: a randomized, placebo-controlled, double-blind, cross-over study with molecular and structural neuroimaging. Int J Neuropsychopharmacol. 2014;18(4):pyu063. doi: 10.1093/ijnp/pyu063

33. Harada $\mathrm{H}$, Kashiwadani $\mathrm{H}$, Kanmura Y, Kuwaki T. Linalool odor-induced anxiolytic effects in mice. Front Behav Neurosci. 2018;12:241. doi: 10.3389/fnbeh.2018.00241 
34. Kim KY, Seo HJ, Min SS, Park M, Seol GH. The effect of 1,8-cineole inhalation on preoperative anxiety: a randomized clinical trial. Evid Based Complement Alternat Med. 2014;2014:820126. doi: 10.1155/2014/820126

35. Lakhan SE, Sheafer H, Tepper D. The effectiveness of aromatherapy in reducing pain: a systematic review and meta-analysis. Pain Res Treat. 2016;2016:8158693. doi: $10.1155 / 2016 / 8158693$
36. Crowley D, Kendall NAS. Development and initial validation of a questionnaire for measuring fear-avoidance associated with pain: the fear-avoidance of pain scale. J Musculoskelet Pain. 1999;7(3):3-19. doi: 10.1300/J094V07N03_02

37. Quinlan-Colwell AD. Understanding the paradox of patient pain and patient satisfaction. J Holist Nurs. 2009;27(3):177-182; quiz 183-185. doi: 10.1177/0898010109332758

This article meets the Accreditation Council for Graduate Medical Education and the American Board of Medical Specialties Maintenance of Certification competencies for Patient Care and Medical Knowledge.

(C2021 by the author(s); licensee Ochsner Journal, Ochsner Clinic Foundation, New Orleans, LA. This article is an open (c) (i) access article distributed under the terms and conditions of the Creative Commons Attribution (CC BY) license (creativecommons.org/licenses/by/4.0/legalcode) that permits unrestricted use, distribution, and reproduction in any medium, provided the original author(s) and source are credited. 